\title{
A before-after analysis for the design problem on an urban road network
}

\author{
G. Pavone ${ }^{2}$, A. Polimeni ${ }^{1} \&$ A. Vitetta ${ }^{1}$ \\ ${ }^{I}$ DIIES - Dipartimento di ingegneria dell'Informazione, \\ delle Infrastrutture e dell'Energia Sostenibile, \\ Università degli Studi Mediterranea di Reggio Calabria, Italy \\ ${ }^{2}$ Regione Calabria, Dipartimento 9, Italy
}

\begin{abstract}
In this paper, a road network design problem at an urban level is reported. After providing a general overview of the problem, a mathematical formulation is provided. In the design model, both the network layout and the signals setting are considered. An application on a real network is performed. In relation to the deign procedure, a comparison between the before and after configurations (generated with a plan or design) is reported. The objective is to increase the safety of road users.
\end{abstract}

Keywords: heuristic algorithms, genetic algorithm, Tabu Search, network constraints.

\section{Introduction}

In the literature, the Network Design Problem (NDP), referred to an urban transportation network, is relative to the network layout and to the signals setting optimization. In the network layout the link directions and the lanes allocation are designed; in the signals setting the phases at junctions and the phases order are designed. A network configuration includes both network layout and signals setting. A first method for classifying the NDPs depends on the design variables considering: the network layout; the signals setting; the network layout and the signals setting. In third case: the layout and the signals setting can be optimized jointly or sequentially; the junctions can be considered isolated or interacting. Generally, the variables involved in network layout are discrete, the variables involved in signals setting are continuous. Considering this, the NDPs can be 
classified also in relation to the variables: discrete, continuous, mixed (with discrete and continuous variables) problems.

Figure 1 shows a general approach to apply for solving a NDP (supply design model). It is a loop approach, which has as input the supply and the demand starting from external objectives and constraints. Mono and multi-objective problems can be considered. The considered case assumes that the demand is rigid. The supply can change at each procedure iteration because it is the control variables (network layout and in signals setting). A demand-supply interaction model allows evaluating the performances and the impacts of actual network configuration. A test is performed to evaluate if the objectives are reached.

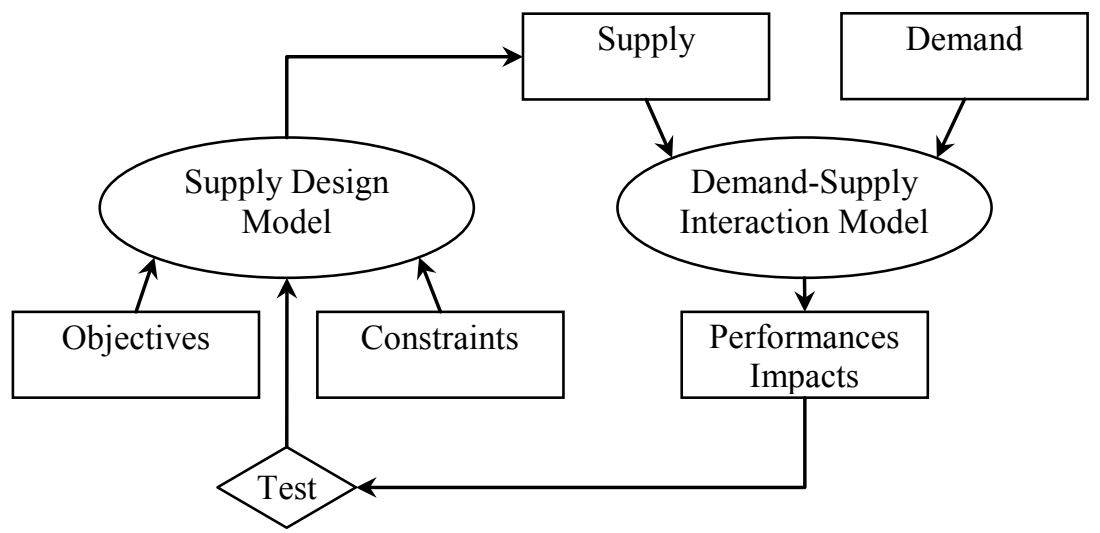

Figure 1: Network Design Problem: a general approach.

Considering the previous classification, Table 1 reports some work present in literature. Some of this work is also briefly commented on in the next paragraphs.

Relating to the network layout Billheimer and Gray [1] propose a heuristic approach to solve the problem in uncongested networks. The problem is extended at congested network (e.g. [2, 3]).

Relating to the signal setting design, two sub-classifications can be made, according to isolated junctions or interacting junctions. In first case, some of these papers are: Webster [20], Webster and Cobb [21], Allsop [8, 9] and Cantarella et al. [12]. In the second case, some of these papers are: Cantarella et al. [12], Sun and Mouskos [28], Marcianò and Vitetta [26]. Other distinctions, based on path choice, can be added.

Relating to the network layout and the signals setting design, Cantarella et al. [11] propose some heuristic approaches to solve the problem considering rigid supply and demand. Poorzahedy and Rouhani [30] propose several hybrid algorithms considering rigid demand and the construction of new lanes. Russo and Vitetta [29] propose a three-step method for the solution selection (topological similarity, cluster analysis, solution selection). Recently the design 
procedures are applied in comparison with the transit design [34]. The models and the procedures and for simulation and design system is applied also in evacuation in urban area $[35,36])$

Table 1: $\quad$ Network Design Problem: design approach.

\begin{tabular}{|c|c|c|}
\hline $\begin{array}{c}\text { Network } \\
\text { layout }\end{array}$ & & $\begin{array}{l}\text { Billheimer and Gray [1], Chen and Alfa [2], Foulds [3], } \\
\text { Poorzahedy and Abulghasemi [4], Solanky et al. [5], Xie and } \\
\text { Turnquist [6], Xu et al. [7] }\end{array}$ \\
\hline \multirow{2}{*}{$\begin{array}{l}\text { Signals } \\
\text { setting }\end{array}$} & $\begin{array}{l}\text { Isolated } \\
\text { junctions }\end{array}$ & $\begin{array}{l}\text { Allsop [8-10], Cantarella et al. [11, 12], Cascetta et al. [13], } \\
\text { Chiou [14], Gartner [15], Meneguzzer [16], Sheffi and } \\
\text { Powell [17], Smith [18], Tan and Gershwin [19], Webster } \\
\text { [20], Webster and Cobbe [21] }\end{array}$ \\
\hline & $\begin{array}{l}\text { Interacting } \\
\text { junctions }\end{array}$ & $\begin{array}{l}\text { Cantarella et al. [12, 22], Ceylan and Bell [23], Gartner [15], } \\
\text { Little [24], Little et al. [25], Marcianò and Vitetta [26], } \\
\text { Marcianò et al. [27], Sun and Mouskos [28] }\end{array}$ \\
\hline $\begin{array}{l}\text { Network } \\
\text { layout } \\
\text { and } \\
\text { signals } \\
\text { setting }\end{array}$ & & $\begin{array}{l}\text { Cantarella et al. [11], Russo and Vitetta [29], Poorzahedy and } \\
\text { Rouhani [30], Gallo et al. [31], Caggiani and Ottomanelli } \\
\text { [32], Polimeni and Vitetta [33] }\end{array}$ \\
\hline
\end{tabular}

In this paper, a formulation of the NDP is proposed and some algorithms to solve it are discussed. Moreover, an analysis to compare the previous configuration (before), the after configurations provided by the urban plan and the designed configuration is reported. The comparison is aimed for testing the quality of the proposed model and procedure and cannot be considered as a plan evaluation. Considering the junction design, integrated with the layout design, the objective is to increase the safety of road users.

The paper is structured as follows. In Section 2, the mathematical formulation of the NDP is reported (considering mono-level, bi-level and equivalent formulation). In Section 3, some algorithms to solve the problem are proposed. In Section 4 an analysis before-plan-design, to compare the network configurations, is reported.

\section{Network design problem}

In this section, a formulation for the NDP is proposed, considering both the network layout and the signals setting. The search for new solutions is influenced by the objectives and the constraints. Some actors, with different objectives, are involved in the problem:

- transport system users, who seek to minimize travel time and monetary costs;

- transport system managers, who seek to maximize the difference between revenues and costs and maximize the safety of the road users; 
- community, who seek both to minimize pollution and maximize usable urban areas.

It is worth noting that these objectives are often conflicting. Moreover, the same individuals usually act as both transport system users and community members, so they themselves have conflicting objectives.

The main kinds of constraints are:

- technical (minimum green times, maximum cycle length, maximum speed, maximum flows, ...);

- external (limit values for concentrations of air pollutants, maximum noise levels, budget constraint, maximum estimated number of accident, ...);

- behavioural (supply-demand interaction, ...).

Decision variables can be classified as:

- integer (for lane allocation, signal stages);

- continuous (for signal settings, signal offsets, road pricing, park pricing);

- mixed integer (for any combination of variables from both the first two sets).

To take into account the conflicting objectives related to the NDP, a multiobjective model could be formulated as in (1).

Objective function: $\quad \mathrm{z}(\mathbf{y}, \mathbf{f})=\left\{\mathrm{z}_{1}(\mathbf{y}, \mathbf{f}) ; \mathrm{z}_{2}(\mathbf{y}, \mathbf{f}) ; \ldots ; \mathrm{z}_{\mathrm{m}}(\mathbf{y}, \mathbf{f})\right\}$

Control variables: $\quad \mathbf{y}$

Constraints:

$$
\begin{aligned}
& \mathbf{y} \in \mathrm{S}_{\mathrm{y}} \\
& \mathbf{f}=\mathbf{f}_{\mathrm{SNL}}(\mathbf{c}(\mathbf{f}, \mathbf{y})) \\
& \mathbf{f} \in \mathrm{S}_{\mathrm{f}}
\end{aligned}
$$

where:

- ${ }^{\mathrm{T}} \mathbf{y}=\left[{ }^{\mathrm{T}} \mathbf{y}^{\mathrm{TO}},{ }^{\mathrm{T}} \mathbf{y}^{\mathrm{SS}}\right]$ is the design variables vector $\left({ }^{\mathrm{T}}\right.$ stays for transpose), with

- $\mathbf{y}^{\text {TO }}$ the vector of variables related to network topology (note that an entry of this vector can be include any elements related to link configuration);

- $\mathbf{y}^{\text {SS }}$ vector of variables related to signals setting (note that an entry of this vector can be include any elements related to the junction configuration);

- $\mathbf{f}$ is the link vector flow;

- $S_{y}$ is the set of admissibility of design variables;

- $S_{f}$ is the set of admissibility of link flow;

- $\mathrm{z}_{\mathrm{i}}$ (with $\left.\mathrm{i}=1,2, \ldots, \mathrm{m}\right)$ is a function representing the $\mathrm{i}^{\text {th }}$ objective;

- $\mathbf{f}_{\mathrm{SNL}}$ stochastic network loading function. 


\subsection{Mono criteria and mono-level formulation}

In mono-criterion and mono-level formulation, the equation (1) is formalized, considering only one objective, as the total travel time spent in the network (5):

$$
\mathrm{z}(\mathbf{y}, \mathbf{f})={ }^{\mathrm{T}} \mathbf{c}(\mathbf{y}, \mathbf{f}) \cdot \mathbf{f}
$$

The objective function can be modified considering the users' safety (i.e. inserting the number of accidents) or a weighed sum of different objectives indicator.

The problem can be formulated as a constrained non-linear mono-level problem.

$$
\text { minimize }_{\mathbf{y}}{ }^{\mathrm{T}} \mathbf{c}(\mathbf{y}, \mathbf{f}) \cdot \mathbf{f}
$$

constraints:

- technical

- structural

$$
\mathrm{y} \in \mathrm{S}_{\mathrm{y}}
$$

- connection and signals setting

$$
\begin{array}{ll}
\mathrm{W}_{\mathrm{rs}}<+\infty & \forall r, s \in C \\
\mathbf{y}^{\mathrm{SS}} \in \mathrm{S}_{\mathrm{g}} &
\end{array}
$$

- (behavioural) equilibrium

$$
\begin{gathered}
\mathbf{f}=\mathbf{f}_{\mathrm{SNL}}(\mathbf{c}(\mathbf{f}, \mathbf{y})) \\
\mathbf{f} \in \mathrm{S}_{\mathrm{f}}
\end{gathered}
$$

where

- $\quad N$, the node set;

- $C \subseteq N$, centroid set;

- $w_{r s}$, path cost;

- $S_{g}$ is the set of admissibility of signal setting design variables.

In the technical constraints structural, connection and signals setting constraints are considered. The former concerning the physical characteristics of the links (e.g. maximum lanes number), the latter concerning the existence of at least a path between each origin-destination pair and the regulation at junctions (e.g. maximum green value).

\subsection{Mono criteria and bi-level formulation}

In a bi-level formulation the equilibrium problem is specified as a component of the problem: 
First level

$$
\text { minimize }_{\mathbf{y}}{ }^{\mathrm{T}} \mathbf{c}(\mathbf{y}, \mathbf{f}) \cdot \mathbf{f}
$$

Second level

$$
\mathbf{f}=\mathbf{f}_{\mathrm{UE}}(\mathbf{y})
$$

constraints:

$$
\begin{aligned}
& \mathrm{y} \in \mathrm{S}_{\mathrm{y}} \\
& \mathrm{w}_{\mathrm{rs}}<+\infty \\
& \mathbf{y}^{\mathrm{SS}} \in \mathrm{S}_{\mathrm{g}} \\
& \mathbf{f} \in \mathrm{S}_{\mathrm{f}}
\end{aligned}
$$

$\mathbf{f}_{\mathrm{UE}}$ is a function that, for each network configuration $\mathbf{y}$, gives the user equilibrium flow.

\subsection{Mono criteria and equivalent formulation}

The problem can be also formulated (the equivalent problem has the same solution of the original problem) as a mono-level problem with linear constraints. Equation (18) formalizes the equivalent problem; the constraints are (14-17).

$$
\text { minimize }_{\mathbf{y}} \mathrm{z}\left(\mathbf{y}, \mathbf{f}_{\mathrm{UE}}(\mathbf{y})\right.
$$

\section{Algorithms}

The procedure proposed to solve the NDP consists of two levels: at the first level considering a problem with integer variables, at the second considering a problem with continuous variables.

The integer design problem requires a high computation effort, this forces us to use heuristic approaches. In [11] some heuristics are proposed to solve the problem: Hill Climbing (HC); Simulated Annealing (SA); Tabu Search (TS); Genetic Algorithms (GA).

Also two hybrid algorithms are proposed (so that the second algorithm start from the result obtained from first algorithm): Tabu Search and Genetic Algorithms (TS+GA); Tabu Search and Path Relinking (TS+PR).

In [11] the best solutions are obtained applying the GA and in the rest of this section will be treated the GA.

The GA [37] is a heuristic based on biological mechanisms related to the survival of species. Three are the main structures in the algorithm: the selection (identify the best elements in the population), the crossover (couple some elements in the population), the mutation (introduce an element of variability in any solutions).

The continuous problems can be solved as in [11], by using a projected gradient algorithm. Known the network layout by the solution of integer 
variables problem, the objective function is evaluated with an assignment procedure, where the signals at the junction are designed and the network performances are calculated.

\section{Analysis before-plan-design}

In this section, an application on real network and a comparison among the old configuration (before), the present configuration actuated by a plan (plan) and the designed configuration (design) is reported. The plan is considered as a benchmark regarding the results of the proposed model and procedure.

The town choice for the comparison is Melito di Porto Salvo (Italy). In Table 2 some characteristics of the city are reported. Figure 2 shows the graph of the study area, representing the before configuration of the network.

Table 2: $\quad$ Melito P.S.: some characteristics.

\begin{tabular}{lc}
\hline inhabitants & 11,594 \\
travel numbers (peak hour) [veic/h] & 3893 \\
nodes & 96 \\
links & 189 \\
network length $[\mathrm{km}]$ & 23 \\
signalized junctions & 37 \\
\hline
\end{tabular}

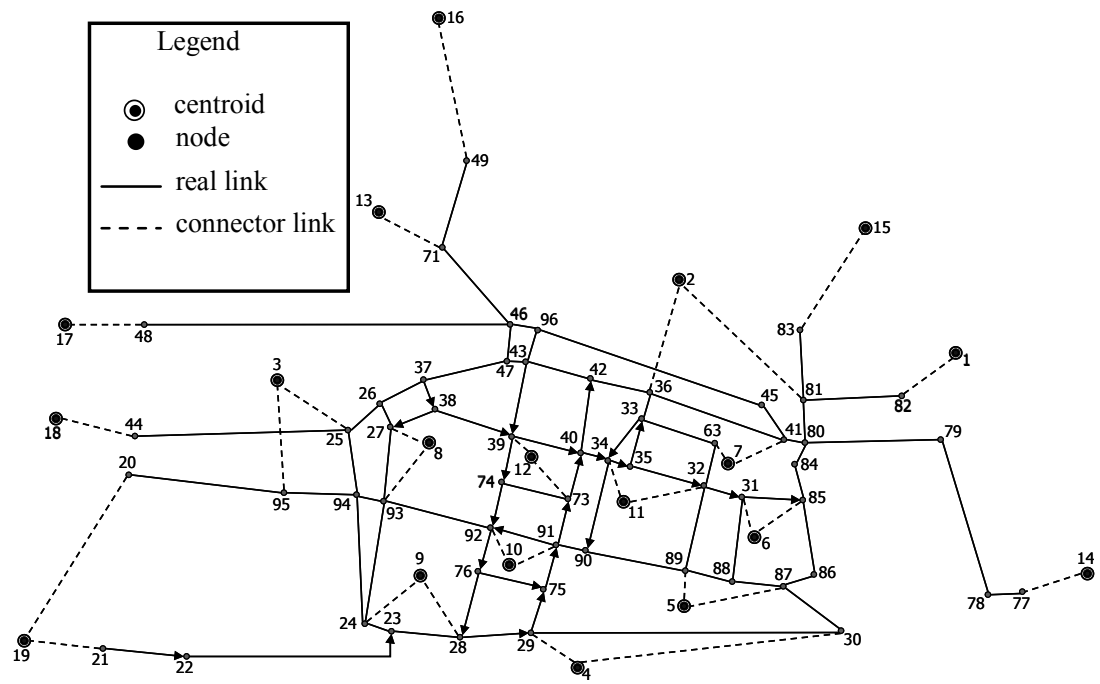

Figure 2: Before network configuration.

The urban traffic plan of Melito P.S. was thinking of encouraging travelling by bus, bicycle and on foot in order to increase safety. This can have to collateral effect the increasing of the total travel time for the car driver. One of the actions 
of the plan is the reduction of lane width, decreasing the link capacity. One of the proposed topologic configurations is reported in Figure 3.

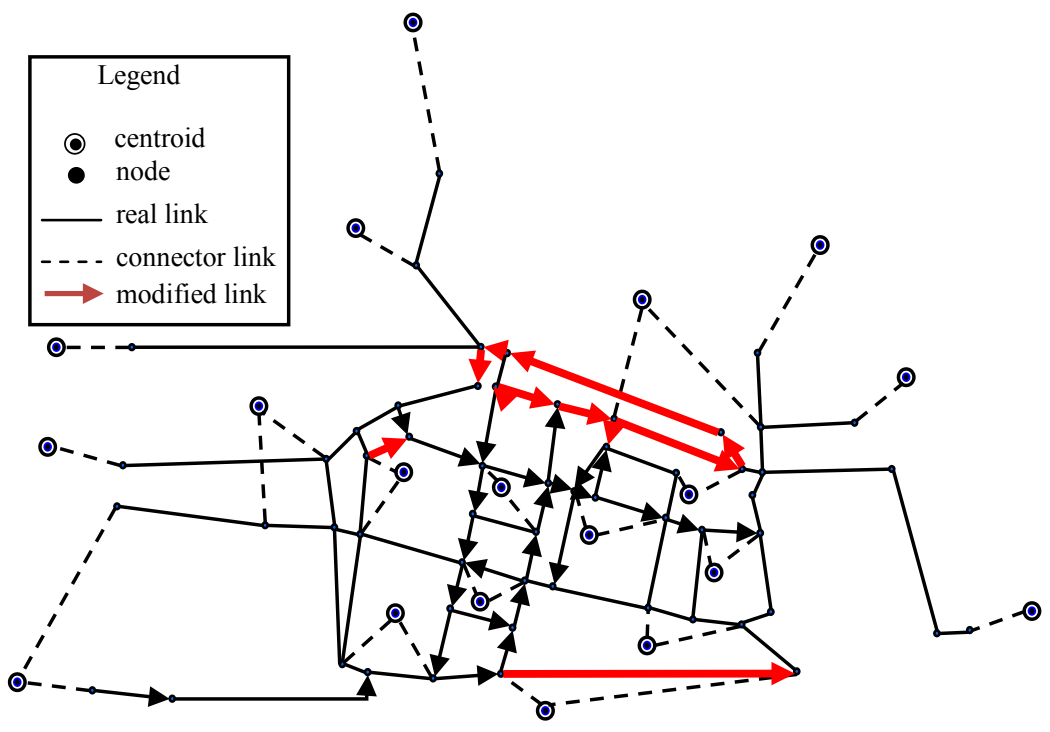

Figure 3: $\quad$ Plan network configuration.

The network design is performed using the formulation reported in Section 2.1. Figure 4 shows the network configuration found with the proposed method.

For comparing the solutions, the $\Delta w$ indicator is defined (note that $\Delta w$ is a negative number when the objective function increases, positive otherwise):

$$
\Delta w=\left(w_{0}-w^{*} / w_{0}\right)
$$

where

$w_{0}$, objective function to minimize value for the before configuration;

$w^{*}$, objective function to minimize value for the plan or design configuration.

Respecting the before configuration, the plan configuration has as variation in the cost the value $\Delta w=-5.7 \%$ (Figure 5). The negative values indicate that the total travel time for car driver increases. It is emphasized that the negative value of $\Delta w$ is caused by the fact that the main objective of the urban plan is to reduce the use of cars.

Comparing the before configuration and the design configuration, the objective function decreases about of $2.1 \%(\Delta w=2,1 \%$, Figure 5$)$. Comparing design configuration with the plan configuration, both the solutions design the main ring as a mono directional way (clockwise in the plan, anticlockwise in the proposed procedure). 


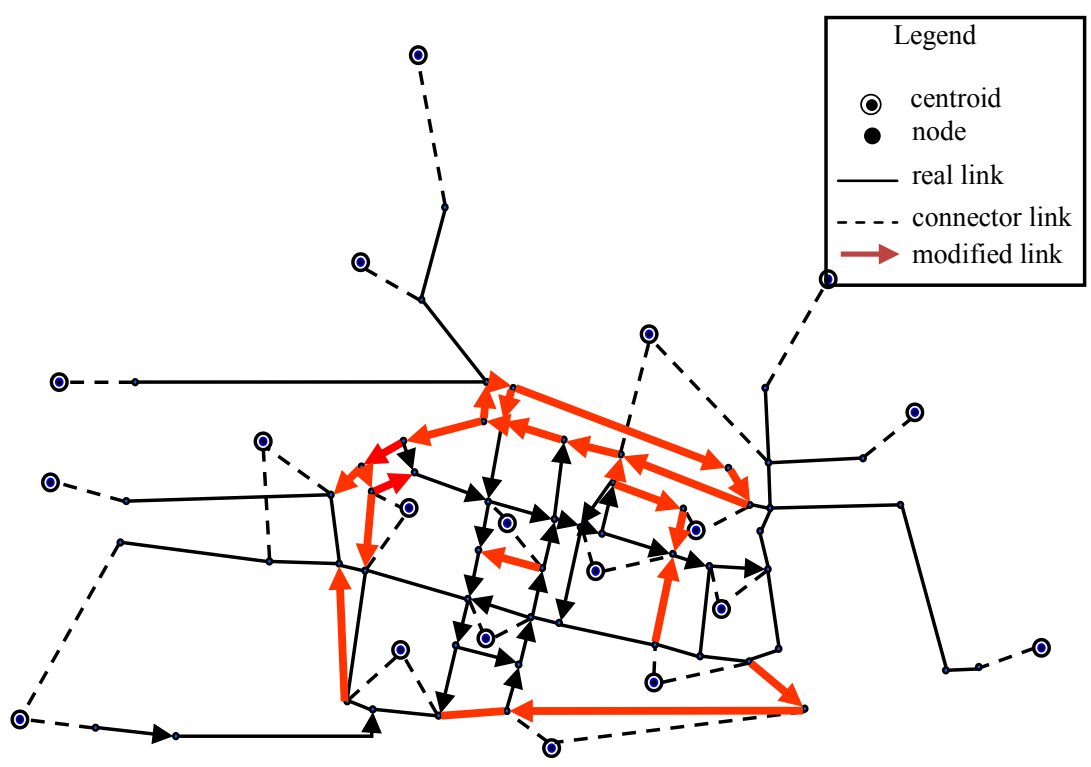

Figure 4: Design network configuration.

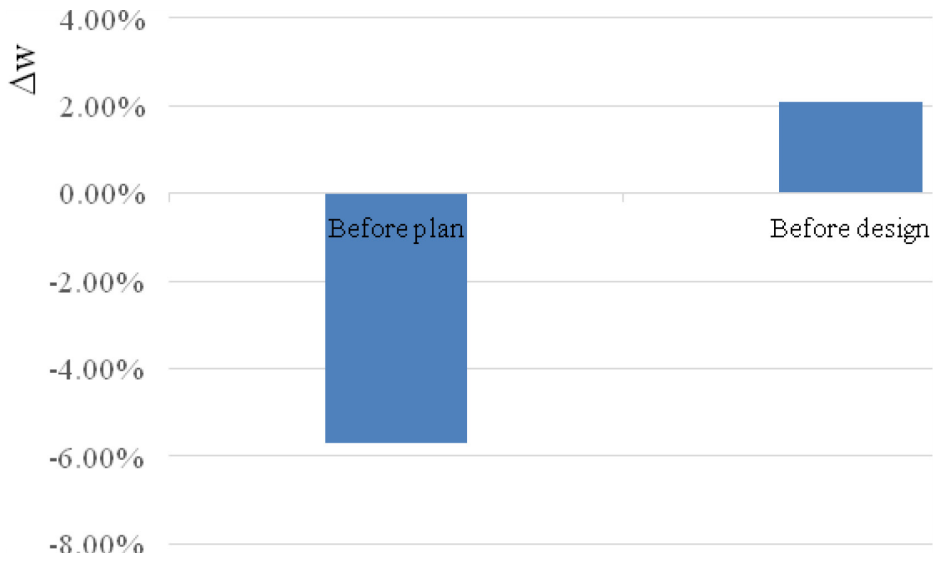

Figure 5: $\quad$ Proposed approach configuration.

\section{Conclusions}

In this paper, the road network design problem is reported. Two variables type are considered: the network layout and the signals setting. The former is approached as a discrete problem, the second as a continuous problem. The solution procedure proposed is heuristic (genetic algorithm). An application in a real case is provided with the aim to compare the plan with the designed network 
configuration, performing a before-plan-after analysis. In detail, three possible configurations are compared: a before configuration, the designed configuration proposed by the urban plan, the designed configuration output of the proposed approach. The comparison is developed defining an indicator related to the comparison of the objective function. From the comparison it emerges that the proposed approach improves the network performances (in terms of road user costs), respect to the plan configuration.

\section{Acknowledgement}

Partially supported by national MIUR under PRIN2009 grants n. 2009EP3S42_001.

\section{References}

[1] Billheimer J.W. and Gray P., Network design with fixed and variable cost elements. Transportation Science, 7, pp. 49-74, 1973.

[2] Chen M. and Alfa, A.S., A network design algorithm using a stochastic incremental traffic assignment approach. Transportation Science, 25(3), pp. 215-224, 1991.

[3] Foulds, L.R., A Multi-Commodity Flow Network Design Problem. Transportation Research Part B 15, 273-283, 1981.

[4] Poorzahedy H. and Abulghasemi F., Application of Ant System to network design problem. Transportation, 32, pp. 251-273, 2005.

[5] Solanky, R.S., J.K. Gorti and Southworth F., Using decomposition in largescale highway network design with a quasi-optimization heuristic. Transportation Research B 32 (2), pp. 127-140, 1998.

[6] Xie C. and Turnquist M.A., Integrated evacuation network optimization and emergency vehicle assignment. TRB, 2009.

[7] Xu T., Wei T., Wang Z.D., Study on continuous network design problem using simulated annealing and genetic algorithm. Expert Systems with Application, 36, pp. 2735-2741, 2009.

[8] Allsop, R.E., SIGSET: A computer program for calculating traffic capacity of signal-controlled road junctions. Traffic Eng. \& Control 12, 58-60, 1971.

[9] Allsop, R.E., SIGCAP: A computer program for assessing the traffic capacity of signal-controlled road junctions. Traffic Engineering \& Control 17, 338-341, 1976.

[10] Allsop R.E., Some possibilities for using traffic control to influence trip destinations and route choice. Sixth Intern. Symposium on Transportation and Traffic Theory. Buckley ed., Amsterdam, Elsevier, pp. 345-374, 1974.

[11] Cantarella G.E., Pavone G. and Vitetta A., Heuristics for urban road network design: lane layout and signal settings. European Journal of Operational Research, 175, pp. 1682-1695, 2006.

[12] Cantarella, G.E., P. Velonà, and Vitetta A., Day-to-day dynamic network modeling and optimization. IEEE International Intelligent Transportation Systems Conference, ITSC 2011; pp. 2086-2092, 2011. 
[13] Cascetta E., Gallo M. and Montella B., Optimal signal setting on traffic networks with stochastic equilibrium assignment. TRISTAN III, 1998.

[14] Chiou S. W., A generalized iterative scheme for network design problem. Applied mathematics and computation, 188, pp. 1115-1123, 2007.

[15] Gartner N.H., Area traffic control and network equilibrium. Traffic Equilibrium Methods, Lecture Notes in Economics and Mathematical Systems, 118, M. Florian, ed., Springer-Verlag, Berlin, pp. 274-297, 1976.

[16] Meneguzzer C., An equilibrium route choice model with explicit treatment of the effect of intersections. Transportation Research B, 29, 329-356, 1995.

[17] Sheffi Y. and Powell W. B., Optimal signal setting over transportation networks. Transportation Engineering, 109(6), pp. 824-839, 1983.

[18] Smith, M.J., The existence, uniqueness and stability of traffic equilibria. Transportation Research B 13(4), 295-304, 1979.

[19] Tan H.N. and Gershwin S.B., Hybrid Optimization: control of traffic networks in equilibrium. LIDS Technical Report, MIT, 1979.

[20] Webster F. W., Traffic signal settings. Road Research Technical Paper no. 39, 1958.

[21] Webster, F.V. and Cobbe B.M., Traffic Signals. Road Research Laboratory Technical Paper 56, London, UK, 1966.

[22] Cantarella G.E., Improta G. and Sforza A., Road network signal setting: equilibrium conditions. Concise Encyclopaedia of Traffic \& Transportation Systems, M. Papageorgiou ed., Pergamon Press, pp. 366-371, 1991.

[23] Ceylan H. and Bell M.G.H., Traffic signal timing optimization based on genetic algorithm approach, including driver's routing. Transportation Research B 38, 329-342, 2004.

[24] Little, J.D.C. (1966) The synchronisation of traffic signals by mixedinteger-linear-programming. Operations Research 14, 568-594.

[25] Little, J.D.C., M.D. Kelson, and N.H. Gartner (1981) MAXBAND: a program for setting signals on arteries and triangular networks. Transportation Research Record 795, 40-46.

[26] Marcianò A. and Vitetta A., A genetic algorithm to solve the traffic signal setting design problem considering driver routing. Proc of XIII Meeting of the Euro Working Group on Transportation, Padova (ITA), 2009.

[27] Marcianò, F.A., G. Musolino, and Vitetta A., Signal setting design on a road network: Application of a system of models in evacuation conditions. WIT Transactions on Information and Communication Technologies, 43 (part I), pp. 443-454, 2010

[28] Sun, W. and Mouskos K.C., Network-wide traffic responsive signal control in urban environments. NCTIP Research Report, New Jersey Institute of Technology, 2002 (source: http://www.transportation.njit.edu/nctip, accessed 26/02/2013).

[29] Russo F. and Vitetta A., A topological method to choose optimal solutions after solving the multi-criteria urban road network design problem. Transportation, 33, pp. 347-370, 2006. 
[30] Poorzahedy H. and Rouhani O.M., Hybrid meta-heuristic algorithms for solving network design problem. European Journal of Operational Research 182(2), pp. 578-596, 2007.

[31] Gallo M., D’Acierno L. and Montella B., A meta-heuristic approach for solving the urban network design problem. European Journal of Operational Research, 201, pp. 144-157, 2010.

[32] Caggiani L. and Ottomanelli M., Traffic equilibrium network design problem under uncertain constraints. Procedia - Social and Behavioral Sciences 20, pp. 372-380, 2011.

[33] Polimeni A. and Vitetta A., Joint network and route optimization in road evacuation. WIT Transactions on Ecology and the Environment, 155, pp. 1053-1065, 2011.

[34] Iannò, D., Polimeni, A., Vitetta, A., An integrated approach for road, transit design in a city logistic plan: A case study. WIT Transactions on the Built Environment 130, pp. 811-822, 2013.

[35] Russo, F., Vitetta, A., A methodology for evacuation design for urban areas: Theoretical aspects and experimentation. Summer Simulation Multiconference, SummerSim 2008, pp. 538-551, 2008.

[36] De Maio, M.L., Musolino, G., Vitetta, A., Traffic assignment models in road evacuation. WIT Transactions on Ecology and the Environment 155, pp. 1041-1051, 2011.

[37] Goldberg D. E., Genetic Algorithms in search, optimization \& machine learning. Addison-Wesley, 1989. 